\title{
Human sperm pattern of movement during chemotactic re-orientation towards a progesterone source
}

\author{
Cecilia Soledad Blengini, Maria Eugenia Teves, Diego Rafael Uñates, Héctor Alejandro Guidobaldi, Laura \\ Virginia Gatica and Laura Cecilia Giojalas
}

\begin{abstract}
Human spermatozoa may chemotactically find out the egg by following an increasing gradient of attractant molecules. Although human spermatozoa have been observed to show several of the physiological characteristics of chemotaxis, the chemotactic pattern of movement has not been easy to describe. However, it is apparent that chemotactic cells may be identified while returning to the attractant source. This study characterizes the pattern of movement of human spermatozoa during chemotactic re-orientation towards a progesterone source, which is a physiological attractant candidate. By means of videomicroscopy and image analysis, a chemotactic pattern of movement was identified as the spermatozoon returned towards the source of a chemotactic concentration of progesterone $\left(10 \mathrm{pmol} \mathrm{I}^{-1}\right)$. First, as a continuation of its original path, the spermatozoon swims away from the progesterone source with linear movement and then turns back with a transitional movement that can be characterized by an increased velocity and decreased linearity. This sperm behaviour may help the spermatozoon to re-orient itself towards a progesterone source and may be used to identify the few cells that are undergoing chemotaxis at a given time.
\end{abstract}

Asian Journal of Andrology (2011) 13, 769-773; doi:10.1038/aja.2011.27; published online 18 July 2011

Keywords: chemotaxis; human spermatozoa; progesterone

\section{INTRODUCTION}

Spermatozoa can find out the egg by following an increasing gradient of attractant molecules. This mechanism is known as sperm chemotaxis. ${ }^{1}$ In the past 20 years, several features of mammalian sperm chemotaxis have been studied, including the size and physiological state of the chemotactic sperm population, the biological sources of attractants, the identity of a physiological attractant candidate, the species specificity of the phenomenon and some signal transduction pathways by which chemotaxis is induced. ${ }^{1}$ Only those spermatozoa that are already capacitated $(\sim 10 \%$ of the cells) are chemotactic at any given time. ${ }^{2-4}$ Follicular fluid, oviductal fluid and the conditioned medium of the egg-cumulus complex are biological sources of chemoattractants. ${ }^{3-11}$ Follicular fluid that has been obtained from one species may attract spermatozoa of other species, suggesting that chemotaxis may not be a species-specific phenomenon. ${ }^{12}$ A small quantity of progesterone, which is secreted by the cells surrounding the egg, is able to attract spermatozoa. ${ }^{7,13-15}$ The transmembrane adenylyl cyclase-cyclic adenosine mono phosphate-protein kinase A pathway and soluble guanylate cyclase-cyclic guanosine monophosphateprotein kinase $G$ pathway, calcium mobilisation and protein Tyrphosphorylation appear to be involved in the chemotactic response mediated by progesterone. ${ }^{14}$

These physiological characteristics of sperm chemotaxis described above, have been primarily observed in human spermatozoa; however, the chemotactic pattern of movement has not been easy to describe. The primary reasons for this are that there are very few chemotactic spermatozoa and that these spermatozoa gradually approximate to the source of the attractant by progressive linear movement. ${ }^{1}$ Nonetheless, in a study where bourgeonal was used as an attractant, a few spermatozoa swam away from the attractant source and then suddenly turned back, apparently owing to an asymmetrical movement of the flagellum. ${ }^{16}$ Interestingly, this sperm behaviour, which has been observed only with bourgeonal, has not been fully described. By contrast, human spermatozoa that have been exposed to a temporal gradient of progesterone show an increase in curvilinear velocity and lateral head displacement, with a decrease in swimming linearity. ${ }^{17}$ However, this sperm behaviour could not be directly related to chemotaxis. Although several molecules have been reported to chemoattract human spermatozoa, ${ }^{1}$ progesterone also seems to be of physiological importance. ${ }^{7,18}$ This study characterizes human spermatozoa movement pattern during chemotactic re-orientation towards a progesterone source. Such a pattern would facilitate the recognition of the few chemotactic-capacitated spermatozoa, which are cells that have the potential to fertilize the egg.

\section{MATERIALS AND METHODS}

\section{Experimental design}

Our hypothesis states that when a spermatozoon senses a gradual decrease in the progesterone concentration (negative gradient), it turns back to the source of the attractant with a specific pattern of movement. In order to identify and characterize such a pattern, we focused this study only on those spermatozoa that turn back to the 
source of the attractant, cells that hereafter, will be referred to as the 'returning' sperm population. In order to guarantee a chemotactic response, a sperm population that contained capacitated spermatozoa, and a physiological attractant $\left(10 \mathrm{pmol}^{-1}\right.$ progesterone $)$ were used to perform the experiments in a chemotaxis chamber. ${ }^{13}$ This chamber had two wells (W1 and W2) that were connected by a bridge over which a capillary space filled with culture medium was formed by adding a coverslip. The cells were loaded into W1, while progesterone was added either to W1 or W2, depending on the experiment. The cells emerging from W1 freely swam over the bridge, while the attractant molecules immediately diffused from the source to the opposite well, forming a unidirectional long-lasting gradient (verification of this gradient was confirmed by using the method of Zigmond ${ }^{19}$ ).

The sperm population that returned to the well that contained progesterone was evaluated in two different settings. First, progesterone was added together with spermatozoa to $\mathrm{W} 1$; hence, the gradient over the bridge was directed from W1 to W2. Because the spermatozoa that were loaded into $\mathrm{W} 1$ were in contact with a homogenous distribution of progesterone, they moved at random inside the well, and some left the well by chance. The emerging sperm population that returned to the well containing the progesterone was analysed (Figure $\mathbf{1 b}$, inset). In a second set of experiments, progesterone was added to W2; thus, the gradient direction was from W2 to W1. Here, the cells first swam from $\mathrm{W} 1$ to $\mathrm{W} 2$, and the cell population returning to W2 was analysed (Figure 1c, inset). In all cases, a chamber that contained a culture medium without progesterone was run in parallel as a negative control. The sperm tracks were video-recorded near the progesterone well (along the bridge), and the corresponding images were analysed as described below. The same semen sample was used for different treatments studied in each experiment.

\section{Sperm preparation}

The experiments were carried out using human spermatozoa in accordance with the guidelines of the Declaration of Helsinki. The semen samples were obtained from healthy donors after 3-5 days of sexual abstinence. Only those samples exhibiting normal seminal parameters according to the WHO criteria ${ }^{20}$ were included in the study. Spermatozoa were separated from the seminal plasma using a discontinuous Percoll gradient ${ }^{21}$ (Sigma-Aldrich, St Louis, MO, USA) with a HAM F-10 culture medium that had been supplemented with $L$-glutamine and $25 \mathrm{mmol} \mathrm{l}^{-1}$ Hepes (Invitrogene, Carlsbad, CA, USA). Next, the highly motile sperm population was adjusted to $6 \times 10^{6}$ cells $\mathrm{ml}^{-1}$ in HAM F-10 that had been supplemented with $1 \%$ human serum albumin (Laboratorio de Hemoderivados, University of Cordoba, Cordoba, Argentina) and then incubated at $37{ }^{\circ} \mathrm{C}$ in $5 \% \mathrm{CO}_{2}$ in air for $4 \mathrm{~h}$, which are conditions that have been established to support sperm capacitation. ${ }^{14}$

\section{Sperm pattern of movement evaluation}

After sperm preparation, the chemotaxis chambers were arranged for each set of experiments, as previously described. ${ }^{3,13-14}$ Briefly, a few drops of culture medium were added over the bridge, and a coverslip was placed upside down on the bridge. First, W2 was filled either with progesterone or the culture medium. Immediately after, spermatozoa were loaded into W1. Progesterone was diluted to a concentration of $10 \mathrm{mmol} \mathrm{l}^{-1}$ in DMSO (Sigma-Aldrich), and the intermediate solutions were prepared with the culture medium getting a $10 \mathrm{pmol}^{-1}$ final concentration of progesterone; hence, the final quantity of the DMSO was negligible, and its addition to the medium did not differ from that of the control group (data not shown). The chemotaxis chambers were incubated for $15 \mathrm{~min}$ at $37^{\circ} \mathrm{C}$, which was the time that was needed for the sperm to redistribute themselves and the progesterone gradient to form over the bridge. Spermatozoa swimming over the bridge were recorded for 3 min under a $\times 4$ objective at $30 \mathrm{~Hz}$ along the border of the progesterone well, and the field was changed each minute. In all treatments, the first recording field was positioned in the middle of the chamber. The videomicroscopy system consisted of a phase microscope (CX41; Olympus, Tokyo, Japan) and a digital camera (ICAM 1500; Labomed, Fremont, CA, USA). The dynamic parameters of the sperm were analysed with two software packages. The ImageJ (NIH, Bethesda, MD, USA) plugin MTrackJ (ver.191.1.0, Eric Meijering; http://www.imagescience.org/meijering/software/mtrackj/) was used to obtain the $\mathrm{X}$ and $\mathrm{Y}$ coordinates of each track, which were then analysed by Spermtrack IV (Centre for Cell and Molecular Biology, University of Cordoba, Argentina) to calculate the sperm kinetic parameters described below.

Qualitative description. The purpose of this research is to identify, in a simple way, a pattern of movement that is representative of chemotaxis; therefore, a qualitative description of the observed patterns was performed. The sperm pattern of movement was qualitatively described with a drawing. Each track consists of two consecutive paths, which correspond to sperm navigation away from and towards the progesterone source. Sperm movement was classified by three researchers on the bases of the lateral head displacement being linear (without visible changes), transitional (with smooth lateral head displacement) or hyperactivated (with a pronounced lateral head displacement). This classification was supported by significant differences in the kinetic parameter values, as shown in Table 1.

Kinetic description. Sperm dynamic changes were evaluated by measuring three sperm parameters: ${ }^{22,23}$ (i) curvilinear velocity (VCL), which is equivalent to the curvilinear distance (DCL) that is travelled by the spermatozoon divided by the time $(\mathrm{VCL}=\mathrm{DCL} / t)$; (ii) linearity (LIN), which is equal to the linear velocity (VSL) divided by the curvilinear velocity ( $\mathrm{LIN}=\mathrm{VSL} / \mathrm{VCL}$ ), wherein VSL is the linear distance divided by the time (LIN values range from 0 to 1 , representing an erratic or straight track, respectively); and (iii) wobble (WOB), which is defined as the average path velocity (VAP) divided by the curvilinear velocity ( $\mathrm{WOB}=\mathrm{VAP} / \mathrm{VCL}$ ) (WOB values vary from 0 (erratic track) to 1 (straight trajectory)). As an internal control, the proportion of chemotactic spermatozoa was determined for each sample, as previously described. ${ }^{3,13,14}$ Only those samples exhibiting a chemotactic response to $10 \mathrm{pmol}^{-1}$ progesterone were included in the study.

\section{Statistical analysis}

In any treatment, the total number of spermatozoa that were analysed in the returning population ranged from 70 to 150 , depending on the sperm sample (e.g., the mean values were 78 and 81 spermatozoa for the control and progesterone treatments, respectively). Three to seven independent experiments were performed with different sperm donors. Statistical differences were determined by one-way ANOVA and Tukey tests using InfoStat software (version 1.1; University of Cordoba).

\section{RESULTS}

Progesterone was first added together with spermatozoa to W1. In the sperm population returning to $\mathrm{W} 1$, six patterns of movement were identified (Figure 1a): 1, linear; 2, transitional; 3, transitional $\rightarrow$ 
a

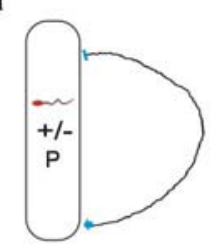

Pattern 1

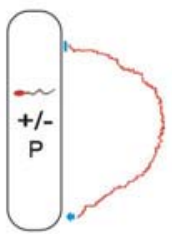

Pattern 2

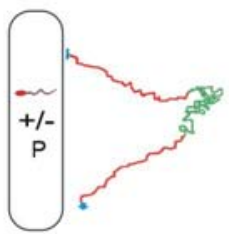

Pattern 3

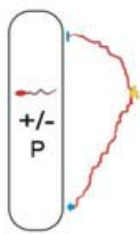

Pattern 4

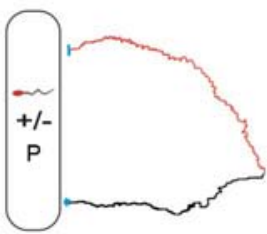

Pattern 5

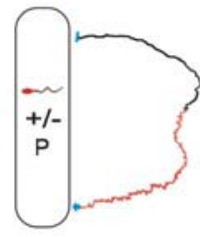

Pattern 6

b
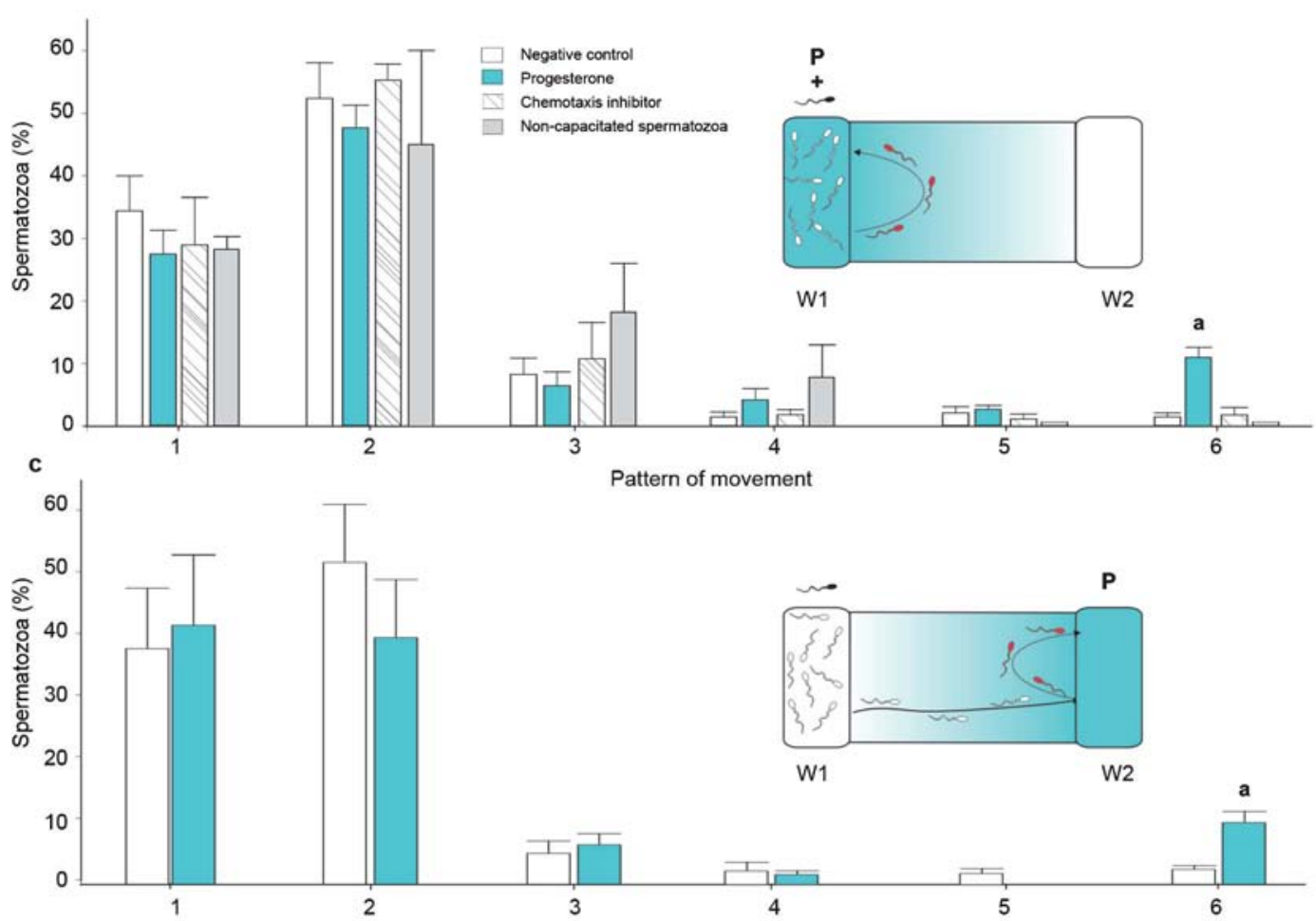

Pattern of movement

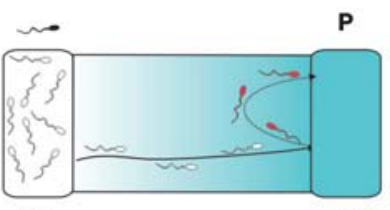

w2

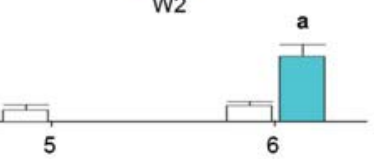

Pattern of movement

Figure 1 Sperm movement patterns during re-orientation to the progesterone source. (a) Qualitative description of the movement patterns that were observed in the sperm population that returned to the well in the absence or presence of progesterone $(P)$; black track: linear; red track: transitional; green track: hyperactivated; yellow track: sperm stop. (b, c) The percentage of spermatozoa that show each of the six patterns of movement described in (a) in two different experimental settings: progesterone was placed in either W1 (b, inset) or W2 (c, inset), wherein the direction of the progesterone gradient is shown in light blue between both wells, and the returning sperm population in red. ${ }^{a} P<0.05$, compared with negative control, chemotaxis inhibitor and non-capacitated spermatozoa. Data are expressed as the mean \pm s.e.

hyperactivated $\rightarrow$ transitional; $\quad 4, \quad$ transitional $\rightarrow$ short $\quad$ stop $\rightarrow$ transitional; 5 , transitional $\rightarrow$ linear; and 6 , linear $\rightarrow$ transitional. Because these patterns were observed both with and without progesterone, the percentage of spermatozoa showing each pattern was calculated for both

Table 1 Kinetic parameters that charactrerized linear, transitional and hyperactivated sperm movement

\begin{tabular}{lrrr}
\hline $\begin{array}{l}\text { Kinetic } \\
\text { parameters }\end{array}$ & Linear & Transitional & Hyperactivated \\
\hline VCL & $27.82 \pm 1.05^{\mathrm{a}}$ & $34.20 \pm 0.89^{\mathrm{b}}$ & $40.32 \pm 1.06^{\mathrm{c}}$ \\
VSL & $25.56 \pm 1.07^{\mathrm{a}}$ & $24.21 \pm 0.75^{\mathrm{a}}$ & $10.23 \pm 1.46^{\mathrm{b}}$ \\
LIN & $0.92 \pm 0.01^{\mathrm{a}}$ & $0.71 \pm 0.02^{\mathrm{b}}$ & $0.49 \pm 0.04^{\mathrm{c}}$ \\
\hline
\end{tabular}

Abbreviations: LIN, linearity; VCL, curvilinear velocity; VSL, linear velocity. Data are expressed as the mean \pm s.e. of 100 spermatozoa. Different superscript letters indicate significant differences compared with other sperm movements $(P<0.05)$. treatments. There was a higher proportion of cells showing pattern ' 6 ' in the presence of progesterone $(P<0.05)$, whereas no differences were observed in the other patterns (Figure 1b). In addition, the size of the sperm subpopulation showing pattern ' 6 ' under progesterone treatment $(10.7 \% \pm 1.4 \%)$ was similar to the proportion of chemotactic spermatozoa $(7 \% \pm 1 \% ; P<0.11)$. In order to verify whether pattern ' 6 ' was associated with a chemotactic behaviour, one of the first steps of the chemotaxis signalling cascade was inhibited. ${ }^{14}$ Thus, before loading the cells into W1, spermatozoa were incubated with an inhibitor of transmembrane adenylyl cyclase (ddAdo, $300 \mu \mathrm{mol} \mathrm{l} \mathrm{l}^{-1}, 15 \mathrm{~min}$; SigmaAldrich), which was previously used by us to specifically inhibit the activity of this enzyme without altering the overall percentage of motile spermatozoa $(>70 \%$ in agreement with previous data shown in the Supplementary Figure 1 in Ref. 14). The percentage of spermatozoa showing pattern ' 6 ' was significantly decreased after this treatment $(P<0.05$; Figure 1b). Because only capacitated cells are capable of chemotactic behaviour, ${ }^{1}$ we carried out experiments by loading noncapacitated cells together with progesterone into W1 as an additional 
negative control. Under this experimental condition, pattern ' 6 ' was not observed when the cells had not previously been capacitated (Figure 1b).

To verify that pattern ' 6 ' was specific to the returning sperm population, the cells were first exposed to a positive gradient of progesterone, which was added to W2 (Figure 1c, inset). Therefore, the cells first had to swim from W1 to W2; those that swam away from W2 and then returned to it were analysed. The same patterns described above were observed (Figure 1a), and the percentage of cells expressing pattern ' 6 ' was significantly higher when progesterone was loaded into the chemotaxis chamber (Figure 1c).

The cells expressing pattern ' 6 ' returned to the progesterone well with transitional movement, which is usually associated with kinetic variations. Therefore, three kinetic parameters were assessed. While swimming away from the progesterone source, pattern ' 6 ' was characterized by a linear movement, whereas the other kinetic parameters remained stable. Conversely, when the sperm cells turned back to the progesterone well, they changed to a transitional movement, wherein the VCL increased and LIN and WOB decreased. These kinetic changes suggest an erratic movement (Supplementary Video 1 and Figure 2).

\section{DISCUSSION}

For sperm to undergo chemotaxis, a concentration gradient of an attractant molecule is required. In species that practice external fertilisation (e.g., marine invertebrates), a dramatic change in the movement of spermatozoa as a function of attractant gradient sensing is easily observed in most of the cells (which change from taking circular paths to helicoid paths). ${ }^{24,25}$ Conversely, in internal-fertilizing species, such as humans, only $\sim 10 \%$ of cells are capable of a chemotactic response while swimming towards the source of an attractant (positive gradient), whereas there is no evidence of a visible change in the pattern of movement. ${ }^{2-4,6,16,26}$ In fact, in our system, the cells that chemotactically swam from W1 (containing spermatozoa) to W2 (containing progesterone) could not be distinguished from the nonchemotactic cells according to the patterns of movement. These features of mammalian sperm chemotaxis make it technically difficult to identify an individual chemotactic cell. Therefore, sperm chemotaxis
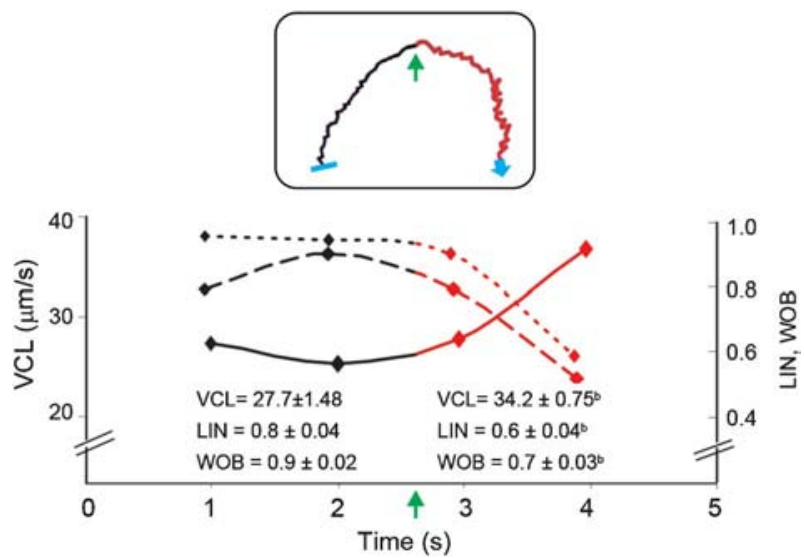

Figure 2 Kinetic parameters of pattern ' 6 ' tracks. A single representative track of pattern ' 6 ' is shown in the inset which curvilinear velocity (VCL, filled line), linearity (LIN, dashed line) and wobble (WOB, dotted line) were determined before (black) and after (red) the cell turned back (green arrow) to the progesterone well and as a function of time. The average values from 27 sperm tracks expressing pattern ' 6 ' are shown in the bottom of the figure, and correspond to both parts of the track (before and after turning) as a function of time. ${ }^{b} P<0.05$, compared with before turning. Data are expressed as the mean \pm s.e. determination is currently limited to a population analysis, and the results are mostly expressed as the percentage of cells oriented towards the attractant source; ${ }^{3,27}$ however, human spermatozoa are able to return to the source of bourgeonal (negative gradient) with asymmetrical movements of the flagella. ${ }^{16}$ This observation directed us to study the sperm behaviour of those cells that return to the source of a physiological attractant candidate, such as progesterone.

Six patterns of movement were identified in the returning sperm population; however, only pattern ' 6 ' seems to be exhibited by cells in the context of sensing a negative attractant gradient. A spermatozoon swimming with pattern ' 6 ' makes a turn towards the progesterone source, and at the same time, the cell movement becomes transitional, with increasing velocity and reduced linearity. These variations in the kinetic parameters are usually observed when the spermatozoon changes from linear to transitional patterns of movement, as shown in Table 1 and also by others. ${ }^{28}$ Regardless of whether progesterone was placed together with spermatozoa or in the opposite well of the chemotaxis chamber, the percentage of cells that exhibited pattern ' 6 ' was higher than among cells in the absence of the hormone or when a chemotactic transduction pathway inhibitor or non-capacitated cells were used.

A similar pattern of movement was observed when human spermatozoa were exposed to a temporal gradient of progesterone ${ }^{17}$ at a concentration range that was several orders of magnitude higher than the picomolar chemotactic concentration. ${ }^{13}$ However, this kind of experiment, which implies a rapid progesterone mixing, does not allow a direct association between the pattern of movement and chemotaxis because the cells cannot detect the attractant source. ${ }^{29}$ In addition, the inhibition of chemotaxis was not included in the experimental design because specific inhibitors were not known at that time. Conversely, pattern ' 6 ' described herein might be related to chemotaxis because (i) the cells are exposed to a temporal-spatial gradient of progesterone, and therefore, the spermatozoon may detect the position of the source of progesterone and orient its movement towards it;

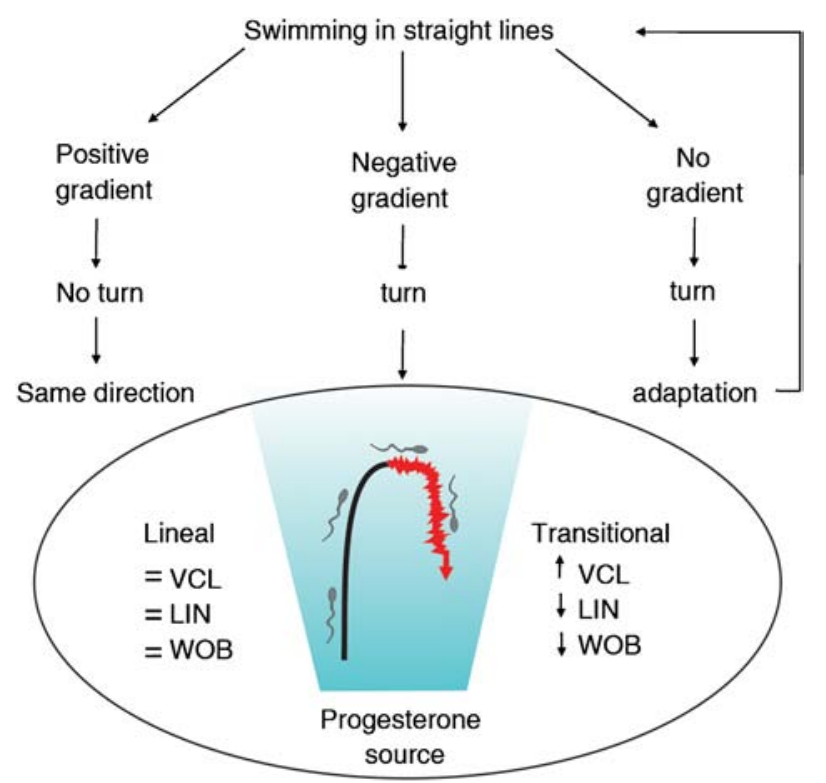

Figure 3 Theoretical model of sperm chemotactic behaviour (adapted with permission from Gakamsky et al. 2009). A representative track of pattern ' 6 ' is included in this model, wherein the linear path is in black and the transitional path is in red, showing the tendency of the kinetic parameters; the attractant gradient is represented in blue (see text for details). 
(ii) the pattern ' 6 ' is expressed by a subpopulation of cells $(\sim 10 \%)$, which is similar to the expected proportion of capacitated-chemotactic spermatozoa; and (iii) when the chemotactic cascade is inhibited or the assays are conducted with non-capacitated spermatozoa, almost no cells are observed to express pattern ' 6 '.

Recently, Gakamsky et al. ${ }^{17}$ proposed an interesting theoretical model to explain the chemotactic behaviour of human spermatozoa that have been exposed to a temporal-spatial attractant gradient. When a spermatozoon swims along the positive gradient towards the attractant source, the cell-surface receptors would be continuously stimulated, and therefore, cell turning would be inhibited. Conversely, when the cell swims away from the source of the attractant (negative gradient) or there is no attractant gradient, the cell changes direction, which increases the chances of relocalizing to the attractant source. Our results experimentally support, in part, the theoretical model described above (Figure 3). Hence, pattern ' 6 ' described herein would correspond to the negative gradient sensing stage that has been proposed in that model; however, Gakamsky et al. ${ }^{17}$ suggested that spermatozoa may not be able to sense a decrease in the chemoattractant concentration since progesterone would irreversibly bind to its receptor because of its low $K_{\mathrm{d}}$ value. Alternatively, the cells might sense a relative change in the attractant concentration, as was recently suggested for sea urchin spermatozoa. ${ }^{30}$

In view of the fact that the chemotactic cells are very few and that a chemotactic marker is not currently available, pattern ' 6 ' might be used to identify chemotactic spermatozoa via observing the returning cell population to a well containing $10 \mathrm{pmol}^{-1}$ progesterone, so as to facilitate further studies of this phenomenon at the molecular level.

\section{AUTHOR CONTRIBUTIONS}

CSB carried out the experiments, analysed the data and participated in the manuscript writing. MET carried out the experiments and participated in the manuscript revision. DRU helped with the setting of the Zigmond chamber experiments. HAG helped with the set-up of the software for sperm tracking. LVG assisted during the experiments. LCG designed the study, obtained financial support and wrote the manuscript.

\section{COMPETING FINANCIAL INTERESTS}

The authors do not have any competing financial interests.

\section{ACKNOWLEDGMENTS}

LCG is a researcher from the National Council for Research (CONICET, Argentina). DRU, MET and HAG are fellowship holders from CONICET. This work received financial support from CONICET (No D918).

Supplementary Information accompanies the paper on Asian journal of Andrology website (http://www.nature.com/aja)

1 Eisenbach M,Giojalas LC. Sperm guidance in mammals - an unpaved road to the egg. Nat Rev Mol Cell Biol 2006; 7: 276-85.

2 Cohen-Dayag A, Tur-Kaspa I, Dor J, Mashiach S, Eisenbach M. Sperm capacitation in humans is transient and correlates with chemotactic responsiveness to follicular factors. Proc Natl Acad Sci USA 1995; 92: 11039-43.
3 Fabro G, Rovasio RA, Civalero S, Frenkel A, Caplan SR et al. Chemotaxis of capacitated rabbit spermatozoa to follicular fluid revealed by a novel directionality- based assay. Biol Reprod 2002; 67: 1565-71.

4 Cohen-Dayag A, Ralt D, Tur-Kaspa I, Manor M, Makler A et al. Sequential acquisition of chemotactic responsiveness by human spermatozoa. Biol Reprod 1994: 50: 78690

5 Gil PI, Guidobaldi HA, Teves ME, Uñates DR, Sanchez R et al. Chemotactic response of frozen-thawed bovine spermatozoa towards follicular fluid. Anim Reprod Sci2008, 108: $236-46$

6 Giojalas LC, Rovasio RA, Fabro G, Gakamsky AM, Eisenbach M. Timing of sperm capacitation appears to be programmed according to egg availability in the female genital tract. Fertil Steril 2004; 82: 247-9.

7 Guidobaldi HA, Teves ME, Uñates DR, Anastasia A, Giojalas LC. Progesterone from the cumulus cells is the sperm chemoattractant secreted by the rabbit oocyte cumulus complex. PLoS One 2008; 3: e3040.

8 Olveira RG, Tomasi L, Rovasio RA, Giojalas LC. Increased velocity and induction of chemotactic response in mouse spermatozoa by follicular and oviductal fluids. J Reprod Fertil 1999; 115: 23-7.

9 Ralt D, Goldenberg M, Fetterolf FP, Thompson D, Dor J et al. Sperm attraction to a follicular factor(s) correlates with human egg fertilizability. Proc Natl Acad Sci USA 1991; 88: 2840-4.

10 Ralt D, Manor M, Cohen-Dayag A, Tur-Kaspa I, Ben-Shlomo I et al. Chemotactic and chemokinesis of human spermatozoa to follicular factors. Biol Reprod 1994; 50: 774-85.

11 Sun F, Bahat A, Gakamsky A, Girsh E, Katz N et al. Human sperm chemotaxis: Both the oocyte and its surrounding cumulus cells secrete sperm chemoattractants. Hum Reprod 2005; 20: 761-7.

12 Sun F, Giojalas LC, Rovasio RA, Tur-Kaspa I, Sanchez R et al. Lack of speciesspecificity in mammalian spem chemotaxis. Dev Biol 2003; 255: 423-7.

13 Teves ME, Barbano F, Guidobaldi HA, Sanchez R, Miska W et al. Progesterone at the picomolar range is a chemoattractant for mammalian spermatozoa. Fertil Steril 2006, 86: 745-9.

14 Teves ME, Guidobaldi HA, Uñates DR, Sanchez R, Miska W et al. Molecular mechanism for human sperm chemotaxis mediated by progesterone. PLoS One 2009; 4: e8211.

15 Teves TE, Guidobaldi HA, Uñates DR, Sanchez R, Miska W et al. Progesterone sperm chemoattraction may be modulated by its corticosteroid-binding globulin carrier protein. Fertil Steril 2010; 93: 2450-2.

16 Spehr M, Schwane K, Riffell JA, Barbour J, Zimmer RK et al. Particulate adenylate cyclase plays a key role in human sperm olfactory receptor-mediated chemotaxis. J Biol Chem 2004; 279: 40194-203.

17 Gakamsky A, Armon L, Eisenbach M. Behavioral response of human spermatozoa to a concentration jump of chemoattractants or intracellular cyclic nucleotides. Hum Reprod 2009; 24: 1152-63.

18 Oren Benaroya, Orvieto R, Gakamsky A, Pnchasov M, Eisenbach M. The sperm chemoattractant secreted from human cumulus cells is progesterone. Hum Reprod 2008; 23: 2339-45.

19 Zigmond HS. Ability of polimorphonuclear leukocytes to orient in gradients of chemotactic factors. J Cell Biol 1977; 75: 606-16.

20 WHO. WHO Laboratory Manual for the Examination of Human Semen and SemenCervical Mucus Interaction. Cambridge: The Press Syndicate of the University of Cambridge; 1987.

21 Aitken RJ, Clarkson JS. Significance of reactive oxygen species and antioxidants in defining the efficacy of preparation techniques. J Androl 1988; 9: 367-76.

22 Davis RO, Siemers RJ. Derivation and reliability of kinematic measures of sperm motion. Reprod Fertil Dev 1995; 7: 857-69.

23 Mortimer ST. A Crtitical review of the physiological importance and analysis of sperm movement in mammals. Hum Reprod 1997; 3: 403-39.

24 Kaupp UB, Hildebrand E, Weyand I. Sperm chemotaxis in marine invertebratesmolecules and mechanism. J Cell Physiol 2006; 208: 487-94.

25 Kaupp UB, Kashikar ND, Weynad I. Mechanism of sperm chemotaxis. Annu Rev Physiol 2008; 70: 93-117

26 Eisenbach M. Mammalian sperm chemotaxis and its association with capacitation. Dev Genet 1999; 25: 87-94.

27 Gakamsky A, Schechtman E, Caplan SR, Eisembach M. Analysis of chemotaxis when the fraction of responsive cells is small-application to mammalian sperm guidance. Int J Dev Biol 2008; 52: 481-7.

28 Mortimer ST, Mortimer D. Kinematics of human spermatozoa incubated under capacitating conditions. J Androl 1990; 11: 195-203.

29 Von Philipsborn A, Bastmayer M. Mechanisms of gradients detection: A comparison of axon path finding with eukaryotic cell migration. Int Rev Cytol 2007; 263: 2-62.

30 Guerrero A, Nishigaki T, Carneiro J, Yoshiro Tatsu, Wood CD et al. Turning sperm chemotaxis by calcium burst timing. Dev Biol 2010; 344: 52-65. 